\title{
Aggregates in silica based matrices
}

O. Cintora-Gonzalez ${ }^{1}$, C. Estournès ${ }^{1}$, J.-L. Guille ${ }^{1 *}$, J.-J. Grob ${ }^{4}$, B. Hönerlage ${ }^{2}$, J. Lemoigne ${ }^{3}$, R. Lévy², T. Lutz ${ }^{1}$, J.-C. Merle ${ }^{2}$, D. Muller ${ }^{4}$, M. Richard ${ }^{1}$, J.-L. Rehspringer ${ }^{1}$, J. Schell ${ }^{2}$ and N. Viart ${ }^{1}$

${ }^{1}$ IPCMS, Groupe des Matériaux Inorganiques, UMR 7504, CNRS-ULP, 23, rue du Loess, 67037 Strasbourg, France

${ }^{2}$ IPCMS, Groupe d'Optique non-linéaire et d'Opto-électronique, UMR 7504, CNRS-ULP, 23, rue du Loess, 67037 Strasbourg, France

${ }^{3}$ IPCMS, Groupe des Matériaux Organiques, UMR 7504, CNRS-ULP, 23, rue du Loess, 67037 Strasbourg, France

${ }^{4}$ Laboratoire PHASE (UPR 292 CNRS), 23, rue du Loess, 67037 Strasbourg, France

Embedding nano-aggregates in silica based supports may be a usefull method to control their size and protect them against degradation. Some examples are given to illustrate the potentiality of this method. Metal aggregates can be obtained on reducing an oxide or salt dispersed in a silica gel or by ion implantation in dense silica layers. In both cases the particles size can be controlled by the conditions of elaboration. Organic nano-crystals such as fullerenes and polydiacetylenes can be also incorporated in mesoporous silica. In this case, the size of the crystals is controled by the size of the pores.

\section{Introduction}

In recent years, nanometer sized particles have attracted much attention, as they present an interest both for fundamental studies and potential applications due to their magnetic or optical properties. These properties, below a critical size, depending on the material and considered property, are size dependent. As a consequence, it is necessary to control the size of the particles and, in numerous cases, to protect them against degradation especially against oxidation. Embedding the particles in an inert matrix can be a suitable way to achieve these goals. Among the possible materials which can be used, silica based ones offer the advantages of versatility, chemical inertness and optical transparency. One of the most ancient examples of such composite materials is the use of noble metals $(\mathrm{Cu}, \mathrm{Ag}, \mathrm{Au})$ colloidal dispersions to get coloured silicate glasses or enamels. More recently, numerous methods have been used to produce these embedded particles. They can be roughly classified as chemical and physical methods. The most often used are, among the first group, ion exchange processes and the sol-gel derived methods and among the second one, evaporation, sputtering and ion implantation. Our purpose is to illustrate the potentiality of some of these methods giving some examples. Two of them are concerned with metal particles and two others with organic crystals.

\section{Metal particles}

\section{Metal particles in silica gels}

One of the most often used chemical method to prepare supported metal particles is the reduction of a metal oxide or salt dispersed in a silica gel (e.g. [1]). Typically, the salt is introduced in a mixture of tetraethyl- (or tetramethyl-) orthosilicate, ethanol and water. After gelification and drying a highly porous xerogel containing the metal in an oxidised state is obtained. Depending on the process used, this xerogel can be obtained in the form of a powder, a film or a monolith. It is subsequently treated in a reducing atmosphere (e.g. hydrogen or hydrogen-argon mixtures). Several different cases have been observed, depending on the nature of the metal. In the case of copper, metal particles are very sensitive to oxidation if the matrix presents an open porosity. Stable particles can only be obtained if, before reduction, a thermal treatment is achieved to close the porosity. After this treatment the gel remains permeable to hydrogen but the permeability to oxygen is drastically reduced. It is then possible to obtain stable metal particles the size of which may be controlled by the temperature of the reduction treatment. The case of nickel is probably the simplest. Stable particles are obtained after every reduction treatment achieved in the $600{ }^{\circ} \mathrm{C}$ to $900{ }^{\circ} \mathrm{C}$ temperature range. Iron and cobalt behave in a more complicated manner as, before being reduced they can form silicates, the reduction of which requires much higher temperatures than the initial salt.

In every case, the resulting average particle size and particle size distribution are strongly dependent on various parameters such as the composition of the initial mixture, the concentration of the reducible species and the thermal treatments. In some cases, the average size may be estimated from X-ray diffraction patterns but the most useful tool is Transmission Electron Microscopy (TEM) which allows a direct measurement of the particle size. It makes possible to correlate the preparation conditions, the particle size and the magnetic (or optical) behavior. As an example, figure 1 presents magnetization versus applied field curves of a nickel silica composite reduced at $600{ }^{\circ} \mathrm{C}$ and $900{ }^{\circ} \mathrm{C}$. The measured average particle sizes are respectively $3 \mathrm{~nm}$ and $10 \mathrm{~nm}$. 


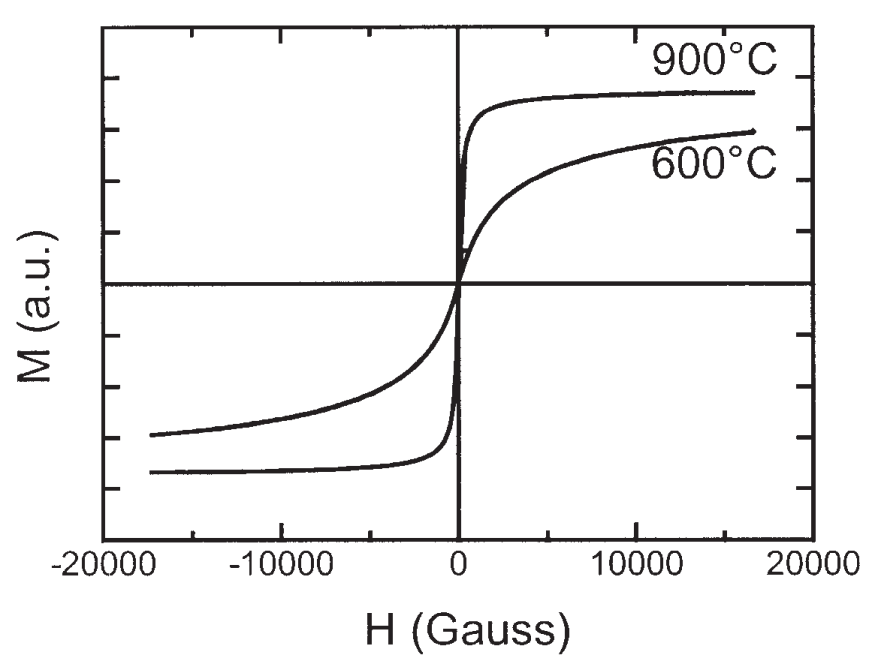

Figure 1. Magnetization versus applied field of two nickel-silica composites.

\section{Metal particles in silica by ion implantation}

Ion implantation is a promising elaboration method to obtain nanoparticles in dense silica based matrices (e.g. [2]). It allows to get metal volume fractions much higher than those attainable by classical melting processes, in a narrow zone close to the surface of the substrate. The metal concentration distribution depends on various parameters, mainly implanted dose, energy of implanted ions, temperature of the substrate, subsequent annealing treatments. The main difference as compared to the previous example is that the total amount of metal is very small (typically $10^{17}$ atoms $/ \mathrm{cm}^{2}$ ) and is heterogeneously distributed in a layer whose width is usually in the order of one hundred nanometers. The most convenient and most often used method to determine the concentration distribution is Rutherford Backscattering Spectroscopy (RBS, see inset). Depending on the chemical nature of both substrate and implanted ions, the latter can remain isolated, react with the matrix and form new compounds, or form metal particles. Once more, MET allows a direct observation of the particles and the determination of their size, size distribution and morphology. Two examples are shown in figure 2 .

The substrate is a silica layer grown by oxydation of a silicon wafer. It was implanted at room temperature with $\mathrm{Co}^{+}$ions $\left(10^{17}\right.$ atoms $\left./ \mathrm{cm}^{2}\right)$ at two incident energies, 30 and $160 \mathrm{keV}$. In both cases RBS has shown that the nickel concentration follows a gaussian distribution.

Increasing the implantation energy makes this distribution to become larger. Correspondingly the mean particle size diminishes. Moreover, it can be seen that it increases progressively from the surface toward the inside then diminishes more abruptly.

Due to the small amount of metal present in these samples, the investigation of the magnetic properties requires the

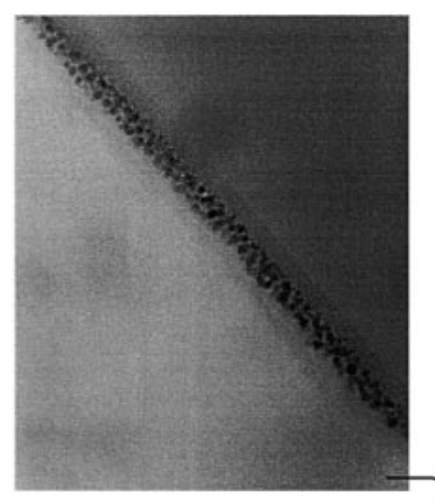

a

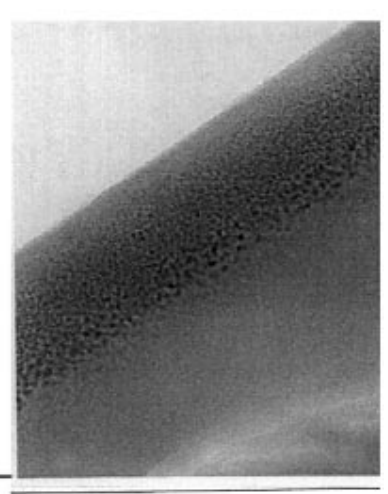

b
$100 \mathrm{~nm}$

Figure 2. TEM micrographs of two Co implanted silica layers (a: $30 \mathrm{keV}, \mathrm{b}: 160 \mathrm{keV}$ ).

use of highly sensitive Superconducting Quantum Interference Device magnetometer (SQUID). The interpretation of the magnetic behavior is by far not straightforward as the particles cannot be considered as isolated and they do not form a continuous film either. However magnetization measurements show that in the as implanted state all the cobalt present is not in the metallic state and that this is only achieved after hydrogen annealing.

\section{Nano-aggregates of organic compounds in mesoporous silica glass for optical purposes}

Due to their structure and composition many organic compounds are optically or electronically active. Carbon-carbon double or triple bonds lead to a strong delocalization of $\pi$ electrons, which can be evidenced by heavy colors or non linear optical phenomena, fluorescence or photoluminescence. Moreover the specific properties of molecules in solution, aggregates, molecular crystals or polymers can enrich the set of molecular states where electron delocalization leads to optical properties. But the study of these properties has to face many practical problems [3]. Theoretically, isolated molecules may be studied in solutions but many problems are often encountered: insufficient solubility, evaporation, bubble formation due to irradiation. Concerning aggregates or nanocrystals, other methods must be found. In this case too, porous silica may be a suitable substrate for these species, but the pore size must be adapted.

\section{Mesoporous glass synthesis and characterization}

Mesoporous glasses are prepared by a sol-gel process. Tetraethoxy silane, $\mathrm{Si}\left(\mathrm{C}_{2} \mathrm{H}_{5} \mathrm{O}\right)_{4}$ (TEOS) in an ethanol solution is reacted with water in presence of a small amount of nitric acid and formamide [4] (1 TEOS, 9 ethanol, 
1 formamide, $5 \mathrm{H}_{2} \mathrm{O}, 0.06 \mathrm{HNO}_{3}$ ). After a first hydrolysis step, polycondensation takes place and gelation occurs in two or three hours depending on the temperature and the additives. Before gelation, the sol is poured in small PMMA boxes to cast the gel in the required size and thickness. The moulded gel is then dried at $40^{\circ} \mathrm{C}$ for $24 \mathrm{~h}$ in a drying oven. A final heat treatment between 500 and $600{ }^{\circ} \mathrm{C}$ is carried out to eliminate the residual solvent (formamide) and to desiccate and strengthen the silica network. In order to increase the porosity of the final product, some additives are introduced in the sol before gelation like voluminous alcohols, for example, who modify the aggregation of silica particles and lead to a large mesoporosity after drying.

This process leads to mesoporous silica pieces approximately $1.5 \mathrm{~cm}$ wide and 0.1 to $1 \mathrm{~mm}$ thick, with a porous volume of $0.29 \mathrm{~cm}^{3} / \mathrm{g}$ and a mean pore size between 3 and $4 \mathrm{~nm}$. These solids have very good optical properties ( 0.01 OD between $210 \mathrm{~nm}$ to $900 \mathrm{~nm}$ ) and can easily be polished to thinner pieces.

\section{Fullerene aggregates in mesoporous silica matrix}

Fullerenes and derived compounds present very interesting optical properties. They exhibit strong non-linearity in the visible range and optical limiting property [5]. But the crystalline material is sensitive to laser beams and sublimates while C60 solutions are useless because, at the laser beams cross point, solvent vapour bubbles occur. Thus, soaking a porous material in saturated solution could be a method to obtain the appropriate optical density in the beams cross point volume. Mesoporous silica pieces have been infiltrated for one hour with a $80{ }^{\circ} \mathrm{C}$ saturated solution of C60 in chlorobenzene and rinsed with a small amount of pure solvent. These samples were used as prepared for optical measurements. When a light beam cross the sample, absorption at $532 \mathrm{~nm}$ increases with the intensity of the incident beam (see inset). The lifetime of excited states depends on the interactions between the molecules and more generally on the interactions with their environment. It can drastically change with the physical state (solution, aggregate or crystal) of the C60 molecules. In solution, where interactions between molecules are weak, the lifetime of S1 lies between 0.6 and $1.2 \mathrm{~ns}$ whereas in crystalline layers it decreases to a few ps. In mesoporous silica, freshly soaked in a $80{ }^{\circ} \mathrm{C}$ saturated solution, an intermediate value of $150 \mathrm{ps}$ is observed, which decreases to $70 \mathrm{ps}$ in aged samples, due to a partial evaporation of the solvent.

\section{Rutherford Backscattering Spectroscopy}

Rutherford Backscattering Spectroscopy (RBS) is a method to analyse the superficial area of materials (less than $1 \mu \mathrm{m}$ ). RBS is quite a simple experiment. A beam of monoenergetic (in the $\mathrm{MeV}$ range) and collimated protons or $\alpha$ particules $\left(\mathrm{H}^{+}, \mathrm{He}^{+}\right)$ impinges on a target and the backscattered fraction of the particles is analysed with a silicon barrier detector placed at the scattering angle.

The two-body elastic scattering between the incident and the target nucleus is the basic concept in backscattering spectroscopy. The energy transfer from the projectile to the target leads to the mass distinction. The probability of collision (scattering cross section), which has been determined for the scattering of protons or $\alpha$ particles on all elements, leads to the capability of quantitative analysis of atomic composition without the need of reference samples. We notice that the scattering cross sections vary as the square of atomic number, therefore RBS is much more sensitive to heavy elements than to light ones. The kinematics of the collision and the scattering cross section are independent of chemical bonding and electronic configuration of the target atoms. Moreover, this technique presents the advantage to be non-destructive.

The projectile atoms, during their trajectory in the matter, loose their energy through interactions mainly with electrons of the sample to be analysed. In a first approximation, nuclear interactions can be neglected in the slowing down of light ions in matter at the energies concerned in RBS (MeV). A good knowledge of the stopping power of the projectile in the analysed material conduct to an estimation of the scattering depth. So we can transform the energy scale to depth scale and therefore deduce the concentration profile. The depth resolution varies with the analysis conditions and the depth, but generally, resolutions as low as $5 \mathrm{~nm}$ can be obtained near the surface in standard conditions.

One of the main applications of RBS is the investigation of ion implantation processes. This technique offers many important informations such as the implantation dose, the concentration profile, the substrate modifications (damages, implantation induced diffusion...). RBS may be very usefully associated with Transmission Electronic Microscopy. An interesting aspect is the comparison between a cross-section TEM image and a profile determined by RBS. We can, in the case of nanoparticules formation and with the help of TEM, quantify the atoms which are diluted in the implanted materials (this quantity equals the solubility limit concentration) and the atoms which contribute to the nanoparticules formation. This measure is particularly important in the case of metallic and magnetic nanoparticules where quantitative measurements of dose and size allow a good understanding of the magnetic behaviour. The part of diluted atoms of $\mathrm{Si}$ or $\mathrm{Ge}$ in implanted $\mathrm{SiO}_{2}$ doesn't contribute to $\mathrm{Si}$ or $\mathrm{Ge}$ nanoparticules formation. The nanoparticule distribution and their evolution can be analysed by RBS and XTEM and some interesting processes have been evidenced by this way (oxidation of diluted atoms, Ostwald ripening...). Some other techniques derived from ion beams like ERDA or nuclear reactions can be useful to determine hydrogen concentration profiles and their evolution during reduction processes of metallic nanoparticles. 


\section{Time-resolved double-pulse pump-probe experiments}

Time resolved pump-probe experiments are a well-known tool of laser-spectroscopy. They are usually used to determine the relaxation dynamics in optically excited materials. Classical time-resolved pump-probe experiments use one strong pump pulse and one corresponding weak test pulse with a variable time delay in between them. The pump pulse excites a number of molecules into an excited state. The test pulse measures the transmission of the sample. Hence, transmission changes due to the presence of the pump pulse-induced excited state population can be detected. The temporal evolution of this excited state population can be monitored if the arrival of the test pulses is delayed.

In most molecules, an excited singlet state is populated by the pump pulse. If the molecules show singlet and triplets excited states, the depopulation of this excited singlet state is either caused by intersystem crossing to the lower-lying triplet state $T_{1}$ or by direct $\mathrm{S}_{1}-\mathrm{S}_{0}$ relaxation. In classical pump-probe experiments only the depopulation due to the combined action of both relaxation processes can be observed, a distinction is not possible. It is not possible, either to determine the value of the triplet quantum yield as long as the absorption cross sections of the excited singlet and triplet states are not known. These parameters are of crucial importance in the description of reverse saturable absorbers, interesting materials for an application in optical power limiting.
However, the triplet quantum yield, the different absorption cross sections as well as the rate constants involved can all be measured simultaneously in a double-pulse pump-probe experiment. The double-pulse pump-probe technique uses two pump pulses of high energy density and one weak test pulse to monitor the transmission changes created by the pump pulses. The first pump pulse excites a certain amount of molecules into the $S_{1}$ state. After a certain time, some of these excited molecules in the $S_{1}$ state undergo intersystem crossing and are found in the long-living triplet state $T_{1}$, the other part undergoes direct $S_{1}-S_{0}$ relaxation. At times much longer than the lifetime of $S_{1}$, the sample contains less molecules in the ground state than before the excitation, a corresponding amount of triplet excited molecules and no more molecules in the $S_{1}$ state.

At this stage a second pump pulse will then be absorbed by this new distribution of molecules in the different states. This second pump pulse will create less change in transmission than the first one. This has two reasons. First, there are less molecules in the ground state and therefore the excitation $S_{0}-S_{1}$ is more difficult. Second, part of the energy of the second pump pulse is absorbed by the molecules in the $T_{1}$ state and excites them further to higher-lying, very short-living triplet states. This energy is dissipated and is therefore lost for the excitation $\mathrm{S}_{0}-\mathrm{S}_{1}$. The difference in the transmission changes, determined experimentally, is used to evaluate the number of molecules in the $T_{1}$ state, and therefore the triplet quantum yield.

\section{Double-pulse pump-probe experiments}

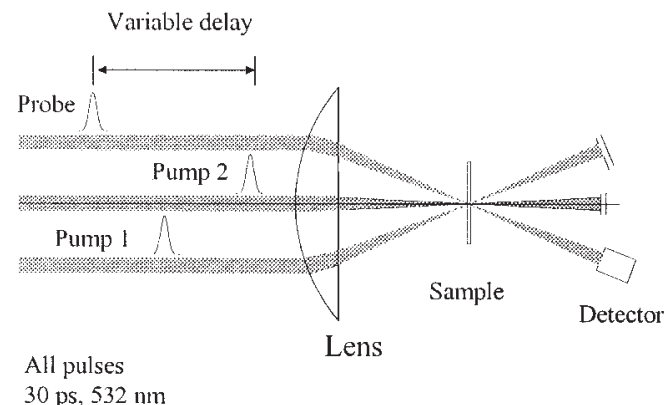

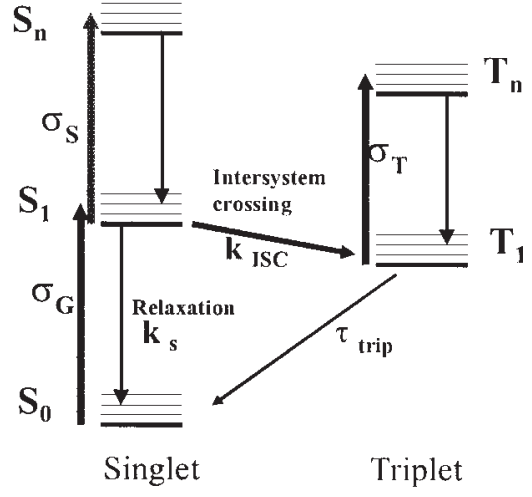

\section{Diacetylene aggregates in mesoporous silica xerogels}

An attempt was made to use the same aggregation process to prepare diacetylene aggregates as precursors for polydiacetylene nanocrystals. The molecule involved in this experiment is the 1,6-bis (9-dicarbazolyl)-2,4- hexadiyne (DCH). This molecule crystallises, in its monomer state, in parallel columns of stacked molecules in the $\mathrm{P} 2_{1} / \mathrm{c}$ space group [6]. Stacking faults are often encountered. When this crystal is heated to $140{ }^{\circ} \mathrm{C}$ triple bonds open and polymerisation occurs between adjacent molecules (Fig. 3).

A long chain of alternate double and single bonds forms and this leads to a drastic colour change: uncoloured monomer crystals become intensely blue poly-DCH crystals with absorption coefficients as high as $10^{7} / \mathrm{mole}$. But the length of the alternate chains of single and double carboncarbon bonds is not regular due to stacking faults in the monomer state. This creates intermediate electronic states, 


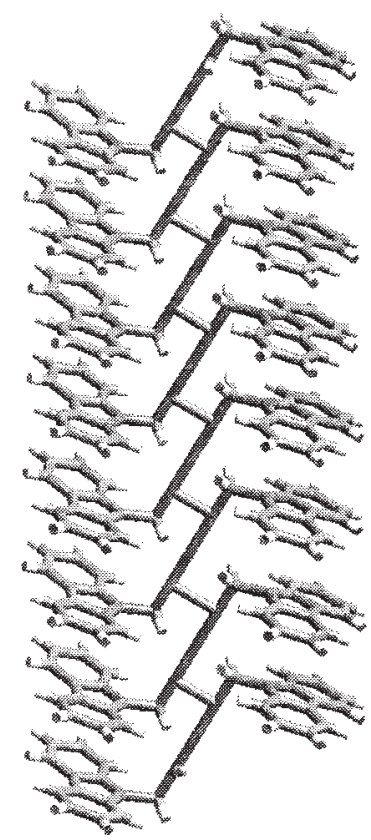

Figure 3.

which contribute to a spectral broadening. Thus spectroscopic results are not easy to explain. The control of the chains length by the porosity of the gels is theoretically conceivable but the solubility of the monomer, whatever the solvent is, is so reduced that it is not possible, using a solution, to bring enough monomer into the pores to form aggregates. Then, another method was used [7]. A suspension of DCH micro-crystals was prepared by pouring a saturated monomer solution in THF. Polymerisation of these particles was induced by U.V. irradiation $(310 \mathrm{~nm})$ and the suspension was ultra-filtrated at $0.22 \mu \mathrm{m}$. This suspension was introduced in the sol-gel mixture before gelation. After gelation and drying $\left(100{ }^{\circ} \mathrm{C}\right)$ a deep blue transparent xerogel was obtained. Figure 4 shows the UV-vis. spectra of the submicronic poly-DCH suspension and of the same suspension included in the silica xerogel. The shift to lower energies of the gel spectrum may be caused by stresses appearing during drying.

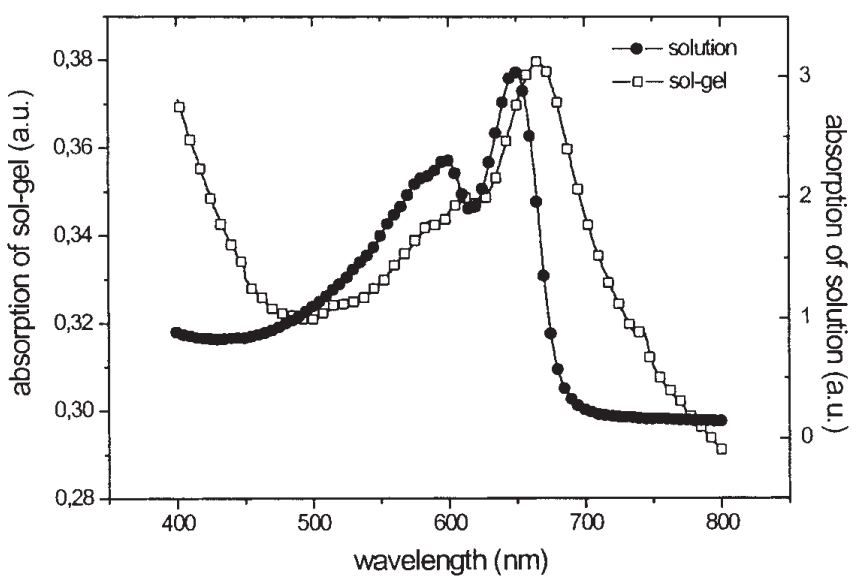

Figure 4.

1. Estournès, C.; Lutz, T., Happich, J.; Quaranta, T.; Wissler, P.; Guille, J.-L. J. Magn. Magn. Mater. 1997, 173, 83-92.

2. Haglund, R. F.; Yang, L.; Magruder, R. H.; White, C. W.; Zuhr, R. A.; Dorsinville, R.; Alfano, R. R. Nucl. Instrum. and Meth. B 1994, 91, 493-504.

3. Chemla, D. S.; Zyss, J. In: Nonlinear otical Properties of Organic Molecules and crystals, Quantum Electronics; Academic Press, 1987.

4. Viart, N. Elaboration par le procédé sol-gel de nanocomposites silice/oxyde de fer magnétique; Thèse de doctorat en science, Université Strasbourg 1, 1997.

5. Levy, R.; Schell, J.; Hönerlage, B.; Rehspringer, J.-L.; Serughetti, J.; Bovier, C. In: Images de la recherche, Les fullérenes; CNRS Éditions: Paris, 1997, pp 44-46.

6. Enkelmann, V.; Schleier, G.; Wegner, G.; Eichele, H.; Schoerer, M. Chem. Phys. Let. 1977, 52, 314-319.

7. Katagi, H.; Kasai, H.; Okada, S.; Oikawa, H.; Matsuda, H.; Nakanishi, H. Pure Appl. Chem. 1997, 10, 2013-2024. 